\title{
Elbow Flexor Strength, Muscle Size, and Moment Arms in Prepubertal Boys and Girls
}

\author{
Louise E. Wood, Sharon Dixon, Chris Grant, \\ and Neil Armstrong
}

\begin{abstract}
The aim of this study was to examine elbow flexion torque, muscle cross-sectional area (CSA), and leverage in boys and girls. Thirty-eight prepubertal children (9.6 \pm 0.3 years) volunteered to participate. All performed isometric flexion actions at $10^{\circ}, 50^{\circ}$, and $90^{\circ}$ of elbow flexion. Magnetic resonance imaging was used to assess elbow flexor (EF) muscle CSA and brachialis moment arm lengths. No significant gender differences were observed for any of the variables studied. EF CSA was directly proportional to isometric torque at $50^{\circ}$ and $90^{\circ}$. CSA explained between $47 \%$ and $57 \%$ of torque variance. Moment arm estimates explained $19 \%$ of the variance in isometric torque at $90^{\circ}$. These baseline data contribute to our understanding of factors influencing strength variation during childhood.
\end{abstract}

Upper-extremity strength differences between boys and girls are of particular interest, given that by adulthood, males outperform females in practically all upper-body sport events involving a strength component (10). Examination of the factors contributing to strength variation will aid our understanding of differences in upper-body performance, but initial baseline data on prepubertal children are required in order to put observations during growth and maturation into context.

The majority of studies have focused on the ability of intrinsic muscle properties to explain strength variation (4); relatively few have examined extrinsic factors, which include the role of anatomy and biomechanics (5). However, unless muscle force is measured directly at the tendon or at the point of application of a load, leverage (an extrinsic factor) will influence measurements of strength. When strength is expressed as joint torque, it represents the product of all the muscle forces and their moment arms contributing to a specified joint action. Given that muscle force is also influenced by moment arm length via its effect on musculotendinous length changes and velocity, it is important to understand the role of variation in moment arm lengths, across a joint range of motion and between individuals, in torque development (15).

\footnotetext{
Wood is with the Department of Sport and Exercise Science, University of Portsmouth, St. Michael's Building, White Swan Road, Portsmouth, PO1 2DT, UK; Dixon is with the School of Sport and Health Sciences, University of Exeter, Exeter, EX1 2LU, UK; Grant is with Somerset MRI Centre, Bridgwater, Somerset, TA6 6LQ, UK; Armstrong is with the Children's Health and Exercise Research Centre, University of Exeter, EX1 2LU, UK, as was Wood at the time of the research.
} 
The musculoskeletal system is considered to act at a mechanical disadvantage, as force or torque measured remote to the muscle tendon will be lower than the actual muscular force generated (4). A more unfavorable mechanical advantage (ratio of muscle moment arm length to skeletal lever arm length) in the presence of unchanging capability of generating force would result in lower measurements of force. Few studies have examined changes in moment arm lengths and therefore mechanical advantage in children in vivo. Most research on upper-extremity moment arms has been conducted on cadavers. However, it is unclear how these data relate to active muscle actions performed by children, especially since changes in muscle size, shape, and surrounding muscles and connective tissues may cause moment arms to vary (8). In adult cadavers, substantial increases in EF moment arm lengths have been observed with elbow flexion (15). In addition, it has been suggested that moment arm lengths may be gender specific (28). A literature search located only two studies $(9,16)$ that have previously examined EF leverage in children. Ikai and Fukunaga (9) studied the biceps brachii mechanical advantage in male subjects, and thus gender comparisons were not possible. Isometric elbow flexion strength and biceps brachii moment arm length were also assessed at only one joint angle $\left(90^{\circ}\right)$. Parker (16) described measurements taken on seven specimens from children's ulna and radius bones to estimate lever ratios. The bone specimens could not be differentiated according to gender, and moment arms could not be estimated across the joint range of motion. The influence of gender and joint angle on mechanical advantage in children therefore remains to be determined.

Muscle moment arms can be estimated using a geometric technique that involves identifying the muscle tendon line of action and the joint center or axis of rotation (2). When estimating muscle moment arm lengths using this method, if the muscle tendon line of action is modeled through the center of the muscle or tendon, increased muscle size will result in greater moment arm lengths (27). Conversely, cadaver studies may substantially underestimate physiological muscle CSAs (11) and therefore moment arms lengths. Changes in muscle size with growth and maturation and muscle size differences between individuals and across genders may alter muscle moment arms. Wretenberg et al. (28) observed significant gender differences in knee muscle moment arms in vivo using magnetic resonance imaging (MRI). Generally, the moment arms of the males were greater than those of the females. The authors made no attempt to explain this finding, but it could be hypothesized that some of the difference may have been the result of the greater muscle mass typically observed in males.

Measurements of upper-extremity muscle size tend to track variation in strength in children during growth and maturation (17). Simultaneous assessment of muscular strength, size, and leverage will enable the contribution of leverage to differences in strength to be discussed. Before examining factors contributing to strength variation after puberty, baseline data should be obtained from prepubertal children. The purpose of this study was therefore to examine upper-extremity leverage and muscle cross-sectional area in prepubertal girls and boys and to assess their contribution to torque development. It was hypothesized that before puberty, there are no significant gender differences in EF muscle moment arms, mechanical advantage, strength, or muscle size across joint angles and that moment arm lengths increase with progressive elbow flexion. 


\section{Methods}

Thirty-eight children (18 boys and 20 girls) volunteered to participate in the study, which was approved by the institutional ethics committee. Written informed consent was obtained from the children and parents or guardians. All children completed strength tests within the Research Centre laboratories. In addition, 17 boys and 16 girls visited a private magnetic resonance center for the determination of muscle size and brachialis moment arm length. The descriptive characteristics of all the participating children are presented in Table 1 and for the children who underwent MR imaging in Table 2. Stature and body mass were measured using a Holtain stadiometer (Crymych, Dyfed, UK) and calibrated beam scales (Weylux, England). Upper-arm length (distance between the superior aspect of the acromion process and the olecranon process) and forearm length (distance between the olecranon process and the styloid process of the radius) were measured using a Harpenden anthropometer configured as a sliding-beam caliper (Holtain, Ltd.). Both limb lengths were measured with the subject standing, and positioned according to the guidelines presented by Martin et al. (14). Pubertal status was assessed during a medical check performed by an experienced school nurse, using Tanner's (22) indices of pubic hair.

\section{Strength Habituation and Tests}

Isometric elbow flexion strength tests were performed on a Biodex System 3 isokinetic dynamometer (Biodex Medical Systems, Shirley, NY) with an adapted seat to accommodate the small limb lengths of the children. The subject's upper arm lay approximately horizontal on a limb support. The axis of rotation of the dynamometer was aligned with the lateral epicondyle of the humerus while the elbow was flexed to approximately $90^{\circ}$. The joint range of motion was set to $100^{\circ} ; 0^{\circ}$ represented elbow extension. The forearm remained in a neutral position for all tests. Only the dominant arm (the limb associated with writing) was examined; all subjects were

\section{Table 1 Descriptive Statistics for 9- to 10-Year-Old Children}

\begin{tabular}{|c|c|c|c|c|c|c|}
\hline & $\mathrm{N}$ & $\begin{array}{c}\text { Age } \\
\text { (y) }\end{array}$ & $\begin{array}{c}\text { Stature } \\
(\mathrm{m})\end{array}$ & $\begin{array}{c}\text { Body } \\
\text { mass } \\
(\mathrm{kg})\end{array}$ & $\begin{array}{c}\text { Arm } \\
\text { lengtha } \\
\text { (cm) }\end{array}$ & $\begin{array}{l}\text { Forearm } \\
\text { length }^{b} \\
\text { (cm) }\end{array}$ \\
\hline Boys & 18 & $\begin{array}{l}9.7 \\
(0.3)\end{array}$ & $\begin{array}{c}1.40 \\
(0.08)^{*}\end{array}$ & $\begin{array}{c}34.2 \\
(10.1)\end{array}$ & $\begin{array}{l}29.0 \\
(1.8)\end{array}$ & $\begin{array}{l}21.1 \\
(1.7)^{* *}\end{array}$ \\
\hline Girls & 20 & $\begin{array}{c}9.5 \\
(0.3)\end{array}$ & $\begin{array}{l}1.35 \\
(0.05)\end{array}$ & $\begin{array}{l}31.7 \\
(5.2)\end{array}$ & $\begin{array}{l}28.1 \\
(1.3)\end{array}$ & $\begin{array}{l}20.2 \\
(0.8)\end{array}$ \\
\hline All children & 38 & $\begin{array}{l}9.6 \\
(0.3)\end{array}$ & $\begin{array}{l}1.38 \\
(0.07)\end{array}$ & $\begin{array}{l}32.9 \\
(7.9)\end{array}$ & $\begin{array}{l}28.5 \\
(1.6)\end{array}$ & $\begin{array}{l}20.6 \\
(1.4)\end{array}$ \\
\hline
\end{tabular}

Note. Values are means (standard deviation).

${ }^{*} p<.05 ;{ }^{* *} p=.05$

${ }^{a}$ Distance between the superior aspect of the acromion process and the olecranon process; ${ }^{b}$ distance between the olecranon process and the styloid process of the radius. 
Table 2 Characteristics for Children Who Underwent Magnetic Resonance Imaging

\begin{tabular}{lcccccc}
\hline & & $\begin{array}{c}\text { Age } \\
\text { (y) }\end{array}$ & $\begin{array}{c}\text { Stature } \\
(\mathbf{m})\end{array}$ & $\begin{array}{c}\text { Body } \\
\text { mass } \\
(\mathbf{k g})\end{array}$ & $\begin{array}{c}\text { Arm } \\
\text { length } \\
(\mathbf{c m})\end{array}$ & $\begin{array}{c}\text { Forearm }^{\mathrm{e}} \\
\text { length } \\
(\mathbf{c m})\end{array}$ \\
\hline Boys & 17 & 9.7 & 1.40 & 34.6 & 28.9 & 21.0 \\
& & $(0.3)$ & $(0.08)$ & $(10.2)$ & $(1.8)$ & $(1.6)$ \\
Girls & 16 & 9.5 & 1.37 & 32.4 & 28.3 & 20.4 \\
& & $(0.3)$ & $(0.04)$ & $(5.5)$ & $(1.3)$ & $(0.7)$ \\
All children & 33 & 9.6 & 1.38 & 33.5 & 28.6 & 20.7 \\
& & $(0.3)$ & $(0.07)$ & $(8.2)$ & $(1.6)$ & $(1.3)$ \\
\hline
\end{tabular}

Note. Values are means (standard deviation).

${ }^{a}$ Distance between the superior aspect of the acromion process and the olecranon process; ${ }^{b}$ distance between the olecranon process and the styloid process of the radius.

identified as right-hand dominant during the process of informed consent. Straps were used to stabilize the shoulders, trunk, hips, and upper arm.

Subjects were familiarized with all of the strength procedures on a separate habituation day. On the test day, a warm-up and a practice period were given before data collection. The strength tests completed were two maximal voluntary isometric flexion actions at $10^{\circ}, 50^{\circ}$, and $90^{\circ}$ of elbow flexion. A $30 \mathrm{~s}$ rest separated successive actions and joint angles, and the order of testing of the three angles was randomized. Subjects were instructed to "pull toward" them and to keep the torque line displayed on the Biodex monitor "as high and as straight as possible." Verbal encouragement was provided throughout. The maximum torque occurring during the "torque plateau" across repetitions was used to represent the isometric torque.

\section{Estimation of Muscle Size and Moment Arms Using Magnetic Resonance Imaging}

For the estimation of muscle size, subjects lay supine with arms resting extended and by their sides, within the whole body core of an MR scanner (Philips 0.5 Tesla Powertrak 1000 system). Ten axial slices (T1 turbo spin echo, echo time $14 \mathrm{ms,}$ repetition time $794 \mathrm{~ms}$, field of view $300 \mathrm{~mm}$, matrix $192 \times 256$, slice thickness $12 \mathrm{~mm}$, slice gap $12 \mathrm{~mm}$ ) were taken along the length of the arm up to the head of the humerus. The initial slice was positioned through the medial and lateral epicondyles of the humerus. The scan time was approximately $2 \mathrm{~min}$.

To examine muscle moment arms, the elbow was fixed in five positions of flexion $\left(10^{\circ}, 30^{\circ}, 50^{\circ}, 70^{\circ}\right.$, and $\left.90^{\circ}\right)$ within the whole body core of the MR scanner. A structure made of nonmetallic materials was designed for this purpose (Figure 1). The subjects lay supine on the base plate with the elbow resting inside a $15 \mathrm{~cm}$ circular receiver coil and aligned with the edge of the vertical plate. The handles were mounted on the vertical plate such that subjects would be required to grasp the handles with the forearm in a neutral position. All the handles were adjustable 
to accommodate different forearm lengths. A strap around the upper arm fixed the position of the humerus throughout the scan sequences. Verbal instructions to grasp each handle firmly and to tense the forearm as if trying to pull the handle toward them were provided before entry into the scanning room. These instructions were reinforced by the radiographers before scanning. Each handle was grasped and sagittal T1 spin echo scan sequences (slice thickness $4 \mathrm{~mm}$, slice gap $0.4 \mathrm{~mm}$, repetition time $393 \mathrm{~ms}$, echo time $25 \mathrm{~ms}$, matrix $192 \times 256$ ) completed.

\section{Analysis}

Muscle Cross-Sectional Area. An infrared mouse and gridded mouse mat were used to trace around the biceps brachii, brachialis, brachioradialis, and pronator teres muscles for each slice. The Philips Gyroview package calculated the area enclosed by each trace. Total EF cross-sectional area was estimated by summing biceps brachialis, brachioradialis, and pronator teres cross-sectional areas in each slice. The maximal total EF cross-sectional area across slices was used to represent the EF cross-sectional area.

Brachialis Moment Arm Lengths. Brachialis muscle moment arm lengths were estimated from the sagittal-plane scans using the geometric method (1). The brachialis EF muscle was chosen because the insertion of the brachioradialis muscle was not imaged, and preliminary observation of the biceps tendon suggested deviation from the sagittal plane. The brachialis tendon has also been reported to lie centrally in the muscle belly (12). Although biceps brachii (long and short heads combined) is typically observed to make the greatest contribution to EF torque (24), brachialis has been deemed to make a similar or even a greater contribution than the biceps to EF torque $(6,11)$. Use of the brachialis for this study can therefore be justified.

The elbow joint center of rotation (COR) was estimated from the sagittal plane scans using the two-dimensional Reuleaux method (13). For the five joint angles examined, application of the Reuleaux method resulted in the estimation

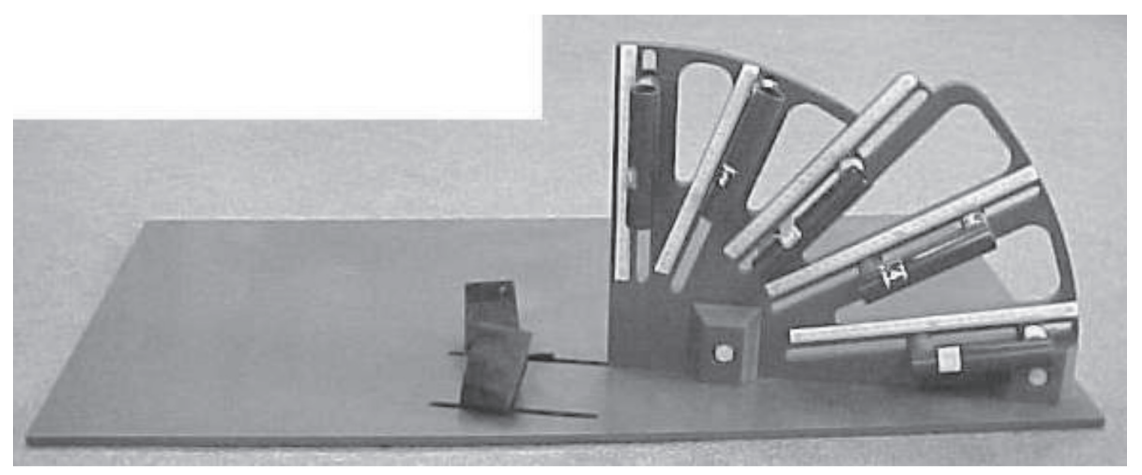

Figure 1-MRI elbow structure designed to fix the elbow in five angles of flexion. 
of four CORs $\left(90^{\circ}-70^{\circ}, 70^{\circ}-50^{\circ}, 50^{\circ}-30^{\circ}, 30^{\circ}-10^{\circ}\right)$. Each COR was estimated six times for each subject based on reliability analyses (detailed later). The COR analysis was performed using the articulation between the trochlea and the trochlear notch of the ulna. The CORs were expressed relative to defined perpendicular axes. The $y$ axis passed through the center of the longitudinal axis of the humerus, and the $x$ axis bisected the trochlea (from superior to inferior). Since there were no significant differences in the COR coordinates across the range of motion or between boys and girls, a mean (fixed) COR coordinate was derived. A nonlinear centroidal approach was then used to model the brachialis muscle-tendon line of action. Coordinates defining the muscle pathway were expressed relative to the same axes used to define the elbow joint center of rotation. The muscle coordinate data were smoothed using Sigmaplot (2000) negative exponential function (Gaussian weighting and a quadratic fit). The lowest-order polynomial and the sampling proportion, which produced smoothed residuals within $0.5 \mathrm{~mm}$ of the entered data, were used (the muscle-tendon coordinates were measured only to the nearest 0.5 $\mathrm{mm})$. The moment arm was estimated as the shortest distance from the smoothed curve to the center of rotation of the elbow using Sigmaplot (2000). The muscle pathway analyses were repeated three times for each subject for each angle (based on reliability analyses). Moment arm lengths were also expressed relative to the skeletal lever arm length to provide an estimate of mechanical advantage (moment arm length/forearm length).

\section{Reliability}

We have previously reported the reliability of the anthropometric, CSA, and isometric torque measures (26). The reliability of the COR and muscle line of action procedures were examined separately for one subject. Ten analyses were completed for this subject's set of scans to examine the reproducibility of the COR positions and the muscle-tendon lines of action. The mean and standard deviation for the $x$ and $y$ COR coordinate positions were calculated after $2,3,4,5,6,7,8,9$, and 10 analyses. These results were used to determine the number of COR analyses performed for all subjects. Similarly, the residuals (difference between the mean moment arm estimated after 10 analyses and after 1 to 9 analyses) were calculated to examine the number of times the muscle-tendon unit needed to be modeled to obtain repeatable moment arm estimates.

\section{Statistical Analysis}

Independent $t$ tests were used to examine gender differences in age, stature, arm length, forearm length, body mass, and muscle CSA. Repeated-measure ANOVA analyses were carried out to examine the significance of differences in isometric flexion torque and brachialis moment arm lengths across angles (repeated measure independent variable) and gender (between-subject independent variable). An alpha of $p<.05$ was deemed significant. If the between-subject gender effect was significant, independent $t$ tests with the Bonferroni adjustment of the significance level were used to determine at which angle significant gender differences were present. 


\section{Assessment of the Contribution of Moment Arms and CSA to Torque}

Allometric scaling was used to assess the relationship between isometric elbow flexion torques and brachialis moment arm lengths at $10^{\circ}, 50^{\circ}$, and $90^{\circ}$ elbow joint angles. The intercept (a) and slope (b) parameters expressed in the linear form of the allometric equation: $\ln \mathrm{Y}=\ln \mathrm{a}+\mathrm{b} \cdot \ln \mathrm{X}$ were solved using ANCOVA (25). Estimates of effect size (eta) were examined to highlight the variance in isometric torque (at $10^{\circ}, 50^{\circ}$, and $90^{\circ}$ ) explained by the variance in moment arm length. Although CSA was measured only with the elbow in an extended position, its relation to joint torque across the joint range of motion was also similarly examined. This allowed the proportionality and potential contribution of muscle size to torque to be examined for boys and girls across angles. Every allometric model was checked for potential misspecification according to Batterham and George (3).

\section{Results}

\section{Descriptive Statistics}

Table 1 illustrates the age and anthropometric characteristics of the 9- to 10-year-old children who completed the strength tests. All children were stage 1 for pubic hair development according to Tanner's (22) indices. The boys were significantly taller than the girls and the difference in forearm length bordered on significant $(p=.05)$. There were no significant gender differences in any of the other variables. Table 2 presents the characteristics of the subgroup who underwent MR imaging.

\section{Isometric Elbow Flexion Torque, Muscle CSA, and Moment Arms}

Mean isometric elbow flexion torques at $10^{\circ}, 50^{\circ}$, and $90^{\circ}$ for boys and girls are presented in Figure 2. Joint angle significantly affected the measured flexion torques; joint torque increased with elbow flexion. Differences in isometric torque across the range of motion were similar for both boys and girls, as illustrated by nonsignificant Angle $\times$ Gender effects. Gender did not significantly affect EF muscle CSA or the torque at each angle. Brachialis muscle moment arm estimates for boys and girls at $10^{\circ}, 30^{\circ}, 50^{\circ}, 70^{\circ}$, and $90^{\circ}$ of elbow flexion are presented in Figure 3. No significant gender differences in moment arm length (Figure 3) were observed. Moment arm lengths differed significantly across the joint angles studied, and these differences were similar for both boys and girls (nonsignificant Gender $\times$ Angle interaction). The largest difference in moment arm length occurred between the $90^{\circ}$ and the $10^{\circ}$ joint positions. This mean difference was $6 \mathrm{~mm}$. When the brachialis moment arm lengths were used to estimate mechanical advantage (Table 3), the mechanical advantage increased with progressive elbow flexion. There were no significant gender differences in mechanical advantage across the range of motion.

The allometric analyses indicated that the moment arm estimates explained $19 \%$ of the variance in isometric torque at $90^{\circ}$. In the more extended joint positions, the moment arm lengths explained less than $1 \%$ of the variance in torque. 


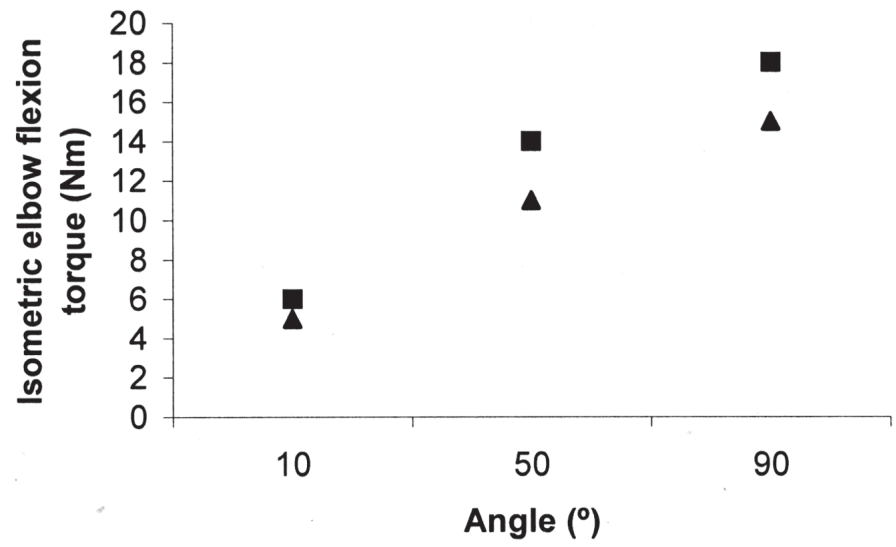

Figure 2-Isometric elbow flexion torques at $10^{\circ}, 50^{\circ}$, and $90^{\circ}$ of elbow flexion for boys (घ) and girls $(\boldsymbol{\Delta})$.

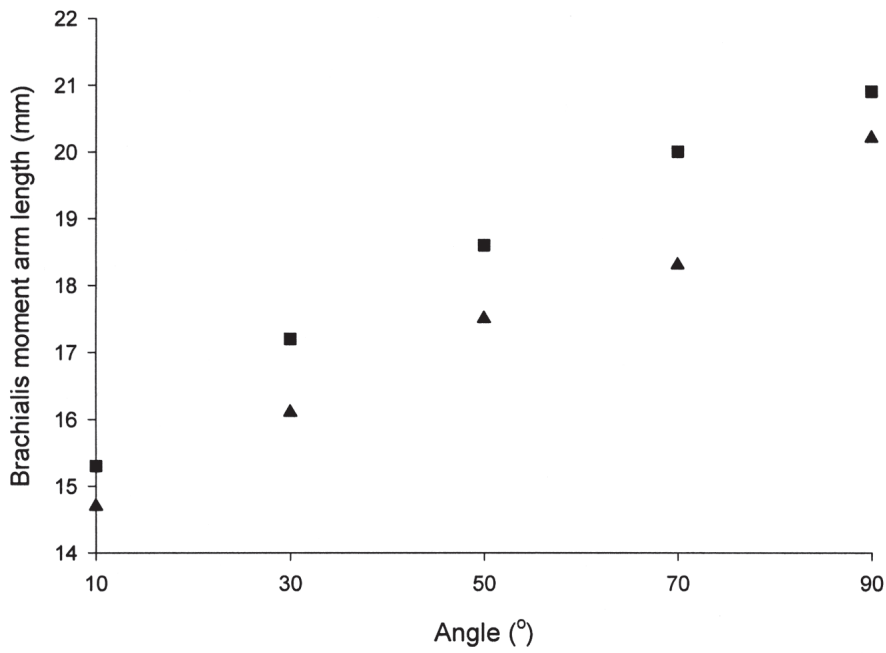

Figure 3-Brachialis moment arm length at $10^{\circ}, 30^{\circ}, 50^{\circ}, 70^{\circ}$, and $90^{\circ}$ of elbow flexion in 9- to 10-year-old boys ( $\square)$ and girls ( $\mathbf{\Lambda})$.

EF CSA was directly proportional to the isometric torque measures at $50^{\circ}$ and $90^{\circ}$ (b exponents of 1.2 and 1.1, respectively). At $10^{\circ}$, the b exponent for CSA was 3.6. The amount of variance in torque explained by the muscle CSA estimates ranged from $43 \%$ to $57 \%$. 
Table 3 Mechanical Advantage of the Brachialis Muscle Over the Joint Range of Motion in 9- to 10-Year-Old Boys and Girls

\begin{tabular}{lccccc}
\hline & \multicolumn{5}{c}{ Mechanical advantage at elbow flexion angles } \\
\cline { 2 - 6 } & $\mathbf{1 0 ^ { \circ }}$ & $\mathbf{3 0 ^ { \circ }}$ & $\mathbf{5 0 ^ { \circ }}$ & $\mathbf{7 0 ^ { \circ }}$ & $\mathbf{9 0}^{\circ}$ \\
\hline Boys & 0.074 & 0.082 & 0.088 & 0.096 & 0.100 \\
& $(0.013)$ & $(0.010)$ & $(0.013)$ & $(0.011)$ & $(0.011)$ \\
Girls & 0.071 & 0.078 & 0.085 & 0.089 & 0.099 \\
& $(0.008)$ & $(0.009)$ & $(0.005)$ & $(0.008)$ & $(0.009)$ \\
All children & 0.073 & 0.081 & 0.087 & 0.093 & 0.100 \\
& $(0.011)$ & $(0.010)$ & $(0.010)$ & $(0.010)$ & $(0.010)$ \\
\hline
\end{tabular}

Note. Values are mean (standard deviation).

\section{Reliability}

After 10 analyses, the minimum and maximum variation (standard deviation) of any of the four CORs along the $x$ axis was $1.3 \mathrm{~mm}$ and $1.8 \mathrm{~mm}$, respectively, and along the $y$ axis $1.0 \mathrm{~mm}$ and $4.2 \mathrm{~mm}$. Trends in the data over the 10 trials were observed to identify the number of analyses required to obtain reproducible COR positions. For the $x$ and $y$ coordinates the residuals generally stabilized after four trials. However, five or six trials were required to obtain consistent positions for the $10^{\circ}$ to $30^{\circ} \mathrm{COR} y$ coordinate and the $50^{\circ}$ to $70^{\circ} \mathrm{COR}$ y coordinate, respectively. Based on these results, six analyses were performed to obtain a COR position and variation representative of that obtained after 10 analyses (and to subsequently obtain the fixed COR coordinate). The maximum error observed across any of the CORs was $\pm 1.9 \mathrm{~mm}(x)$ and $\pm 5.5 \mathrm{~mm}(y)$ after six analyses. The $x$ and $y$ positions of the COR coordinates were more reliable for $90^{\circ}$ to $50^{\circ}$ elbow flexion compared to $50^{\circ}$ to $10^{\circ}$ elbow flexion.

Variation in moment arm estimates due to modeling of the muscle-tendon tended to be below that expected due to the resolution of measurement, which was $0.3 \mathrm{~mm}$ (the resolution reflects the scale of the scans during analysis). This was true after three analyses. Therefore, three trials were performed to estimate moment arm length at each joint angle for all participants. The standard deviation of the moment arm estimates after three trials for each joint angle were $0.2 \mathrm{~mm}$ $\left(90^{\circ}\right), 0.1 \mathrm{~mm}\left(70^{\circ}\right), 0.0 \mathrm{~mm}\left(50^{\circ}\right), 0.1 \mathrm{~mm}\left(30^{\circ}\right)$, and $0.2 \mathrm{~mm}\left(10^{\circ}\right)$.

\section{Discussion}

There were no significant gender differences in isometric elbow flexion torques or CSAs in the present study. These results are in agreement with the extant literature, which suggests that although on average, boys may have slightly larger muscles and perform slightly better in muscle function tests than girls in early childhood, these differences are generally not significant $(17,23)$. Similarly, there were no significant gender differences in brachialis moment arm lengths or mechanical advantage across 
the range of motion examined. The mean mechanical advantage across angles (boys and girls combined) ranged from 0.073 to 0.100 , which is comparable to the ulna (brachialis) lever ratios of between 0.069 and 0.132 presented for bone specimens (which could not be differentiated according to gender) by Parker (16). The present study is the first to examine moment arm length and mechanical advantage across the range of motion for both genders in prepubertal children.

Brachialis moment arm lengths followed a similar pattern to joint torque across the range of motion -increasing with progressive joint flexion and peaking at angles exceeding $75^{\circ}(15)$. The mean peak moment arm length of approximately $20.5 \mathrm{~mm}$ (at $90^{\circ}$ elbow flexion) was similar or lower than moment arms determined in adult cadavers. For example, maximum moment arm length was $21 \mathrm{~mm}$ in An et al. (1) but approximately $30 \mathrm{~mm}$ in Ettema et al. (8), Pigeon et al. (18), and van Bolhuis and Gielen (24). Given the inherent limitations of applying cadaver data to in vivo muscle-tendon properties, these findings suggest the potential for development as growth and maturation progress. This is also indicated by the absolute and relative change in brachialis moment arm length across the range of motion examined. The mean moment arm length of the boys and girls combined varied by approximately $6 \mathrm{~mm}$ between $10^{\circ}$ and $90^{\circ}$ elbow flexion. This absolute difference in moment arm length is less than the $25 \mathrm{~mm}$ and $16 \mathrm{~mm}$ difference presented by Pigeon et al. (18) and An et al. (1), respectively, over a similar range of motion in adult cadavers. Expressed as a percentage of change in moment arm length (maximum-minimum/maximum), the $6 \mathrm{~mm}$ difference across the range of motion in the children was 27\%. Murray et al. (15) estimated a $32 \%$ and $58 \%$ change in brachialis moment arm length in female and male cadavers, respectively, across a slightly different range of motion $\left(25^{\circ}\right.$ to $\left.110^{\circ}\right)$. These moment arm length changes may reflect the shorter limb and muscle lengths of the children, since for a given joint angular displacement, a shorter muscle length will undergo a smaller length change (26).

Elbow flexor CSA was directly proportional to isometric torque at $50^{\circ}$ and $90^{\circ}$; CSA explained 43 to $57 \%$ of torque variance across the whole joint range of motion. This is in agreement with Enoka (7), who stated that approximately 50\% of strength differences are explained by differences in muscle size. Despite the fact that the contribution of moment arm length to torque variance was assessed using only the brachialis moment arm length (which has a small moment arm length relative to biceps brachii and brachioradialis), approximately $19 \%$ of the variance in isometric torque at $90^{\circ}$ was explained. However, the moment length was limited in its ability to explain virtually any of the torque variance at $10^{\circ}$ or $50^{\circ}$. The superior predictive ability at $90^{\circ}$ may be explained by the fact that when the elbow is flexed beyond $75^{\circ}$ or $80^{\circ}$, the moment arms of the flexors are nearing their optimal lengths $(15,18)$. Also in more extended joint positions, the force produced by the flexors is directed more into compressing the joint than causing joint rotation. The same reasoning may (in part) account for the lack of a directly proportional relationship between isometric elbow flexion torque at $10^{\circ}$ and CSA. It should be emphasized that brachialis moment arm length in this study was estimated to explore strength variation in children. It is, however, acknowledged that the brachialis muscle has a broad origin and insertion, and variation in muscle fiber length and orientation within the muscle has led some authors to estimate moment arms for different regions of this muscle (8). The moment arm lengths of the other EF muscles, the impact of differences in architecture (within and between muscles), as well as other 
intrinsic and extrinsic factors (for example, muscle activation and muscle-tendon stiffness) need to be explored in relation to the unexplained variance in torque. In contrast to the strength measurements, the elbow flexors were not maximally activated during the MRI procedures; this would reduce the variance explained by the muscle-tendon properties in the current study.

Any discussion of the meaning of variation in moment arm length should take into consideration measurement reliability. The estimation of moment arms using the geometric technique highlighted that the error due to placement of the COR far exceeded the error due to the muscle line of action. The COR measurement errors obtained after six analyses were similar to those reported in other studies for a variety of joints $(19,20,21)$. However, these errors must be considered in relation to the impact on moment arm estimation for the elbow. The mean moment arm of the boys and girls at $90^{\circ}$ was $20.6 \mathrm{~mm}$ and at $10^{\circ}$ was $15.0 \mathrm{~mm}$. A sensitivity analysis was performed to assess the effect of moving the COR by $\pm 1.9 \mathrm{~mm}$ along the $x$ axis and $\pm 5.5 \mathrm{~mm}$ along the $y$ axis in one child (who had average moment arms similar to the mean group data) for the $90^{\circ}$ and $10^{\circ}$ elbow positions. Displacement of the COR along the $x$ axis for $90^{\circ}$ of flexion resulted in the moment arm estimation differing by $-0.7 \mathrm{~mm}$ to $+1 \mathrm{~mm}$ ( $8 \%$ error). Displacement of the COR along the $y$ axis for the flexed joint position yielded error in the moment arm estimates of $-1.2 \mathrm{~mm}$ to $+1.6 \mathrm{~mm}$ ( $13.6 \%$ error). With the elbow in only $10^{\circ}$ of flexion the equivalent errors along the $x$ and $y$ axes were $\pm 1 \mathrm{~mm}(6.7 \%$ error $)$ and $-0.1 \mathrm{~mm}$ to $0 \mathrm{~mm}(0.7 \%$ error), respectively. These errors must be considered when interpreting the moment arm differences (between boys and girls and across the range of motion) observed in the prepubertal children in the present study.

In conclusion, in 9- to 10-year-old prepubertal children, there were no significant gender differences in elbow flexion isometric torques, muscle CSAs, brachialis moment arm lengths, or mechanical advantage. Brachialis moment arm length contributed to the explanation of some of the variance in isometric elbow flexion torque at $90^{\circ}$ but very little in more extended positions. Changes in the brachialis moment arm length across the joint range of motion followed a similar pattern to that observed in cadavers, although the absolute and relative change in moment arm length was less. Future studies are required to corroborate these findings for the brachialis and other elbow flexor muscles in children. When moment arm data are collected in pubertal children (where gender differences in upper extremity strength have been found to be significant), these baseline data will facilitate understanding of torque development in relation to changes in muscle size and leverage. Estimates of the reliability of isometric torques, muscle size, and moment arm measures in children will clarify the meaning of any differences observed during growth and maturation.

\section{Acknowledgments}

This study was supported by a grant from the Darlington Trust.

\section{References}

1. An, K.N., F.C. Hui, B.F. Morrey, R.L. Linscheid, and E.Y. Chao. Muscles across the elbow joint: a biomechanical analysis. J. Biomech. 14:659-669, 1981. 
2. An, K.N., K. Takahashi, T.P. Harrigan, and E.Y. Chao. Determination of muscle orientations and moment arms. J. Biomech. Eng. 106:280-282, 1984.

3. Batterham, A.M., and K.P. George. Allometric modelling does not determine a dimensionless power function ratio for maximal muscular function. J. Appl. Physiol. 83:2158-2166, 1997.

4. Blimkie, C.J.R. Age- and sex-associated variation in strength during childhood: anthropometric, morphologic, neurologic, biomechanical, endocrinologic, genetic and physical activity correlates. In: Perspectives in Exercise Science and Sports Medicine: Youth, Exercise and Sport, C.V. Gisolfi, and D.R. Lamb (Eds.). Indianapolis: Benchmark, 1989, pp. 99-163.

5. Blimkie, C.J.R., and D. Macauley, Muscle strength. In: Paediatric Exercise Science and Medicine, N. Armstrong, and W. Van Mechelen (Eds.). Oxford: Oxford University Press, 2000, pp. 133-142.

6. Edgerton, V.R., R.R. Roy, and P. Apor. Specific tension of human elbow flexor muscles. In: Biochemistry of Exercise VI: International Series on Sport Sciences, B. Saltin, (Ed.). Champaign, IL: Human Kinetics, 1986, pp. 487-500.

7. Enoka, R.M. Neuromechanical Basis of Kinesiology (2nd ed.). Champaign, IL: Human Kinetics, 1994.

8. Ettema, G.J.C., G. Styles, and V. Kippers. The moment arms of 23 muscle segments of the upper limb with varying elbow and forearm positions: implications for motor control. Hum. Mov. Sci. 17:201-220, 1998.

9. Ikai, M., and T. Fukunaga. Calculation of muscle strength per unit cross-sectional area of human muscle by means of ultrasonic measurement. Int. Z. Angew. Physiol. Einschl. Arbeitsphysiol. 26:26-32, 1968.

10. Jones, D.A., and J.M. Round. Strength and muscle growth. In: Paediatric Exercise Science and Medicine, N. Armstrong, and W. Van Mechelen (Eds.). Oxford: Oxford University Press, 2000, pp. 133-142.

11. Kawakami, Y., K. Nakazawa, T. Fujimoto, D. Nozaki, M. Miyashita, and T. Fukunaga. Specific tension of elbow flexor and extensor muscles based on magnetic resonance imaging. Eur. J. Appl. Physiol. Occup. Physiol. 68:139-147, 1994.

12. Macrander, S.J, The elbow. In: Anatomy and MRI of the Joints. A Multiplanar Atlas, W.D. Middleton, and T.L. Lawson (Eds.). New York: Raven Press, 1989, pp. 49-53.

13. Maganaris, C.N., V. Baltzopoulos, and A.J. Sargeant. Changes in Achilles tendon moment arm from rest to maximum isometric plantarflexion: in vivo observations in man. J. Physiol. 510:977-985, 1998.

14. Martin, A.D., J.E.L. Carter, K.C. Hendy, and R.M. Malina. Segment lengths. In: Anthropometric Standardisation Reference Manual, T.G. Lohman, A.F. Roche, and R. Martorell (Eds.). Champaign, IL: Human Kinetics, 1988, pp. 9-26.

15. Murray, W.M., S.L. Delp, and T.S. Buchanan. Variation of muscle moment arms with elbow and forearm position. J. Biomech. 28:513-525, 1995.

16. Parker, D.F. Factors Controlling the Development of Strength of Human Skeletal Muscle. PhD thesis, University of London, 1989.

17. Parker, D.F., J.M. Round, P. Sacco, and D.A. Jones. A cross-sectional survey of upper and lower limb strength in boys and girls during childhood and adolescence. Ann. Hum. Biol. 17:199-211, 1990.

18. Pigeon, P., L'H. Yahia, and A.G. Feldman. Moment arms and lengths of human upper limb muscles as functions of joint angles. J. Biomech. 29:1365-1370, 1996.

19. Rugg, S.G., R.J. Gregor, B.R. Mandelbaum, and L. Chiu. In vivo moment arm calculations at the ankle using magnetic resonance imaging (MRI). J. Biomech. 23:495-501, 1990.

20. Spoor, C.W., and J.L. Van Leeuwen. Knee muscle moment arms from MRI and from tendon travel. J. Biomech. 25:201-206, 1992. 
21. Stokdijk, M., J. Nagels, and P.M. Rozing. The glenohumeral joint rotation centre in vivo. J. Biomech. 33:1629-1636, 2000.

22. Tanner, J.M. Growth at Adolescence. Oxford: Blackwell Scientific Publications, 1962.

23. Tanner, J.M., P.C.R. Hughes, and R.H. Whitehouse. Radiographically determined widths of bone, muscle and fat in the upper arm and calf from age 3-18 years. Ann. Hum. Biol. 8:495-517, 1981.

24. van Bolhuis, B.M., and C.C.A.M. Gielen. The relative activation of elbow-flexor muscles in isometric flexion and flexion/extensions movements. J. Biomech. 30:803811, 1997.

25. Welsman, J.R., and N. Armstrong. Statistical techniques for interpreting body sizerelated exercise performance during growth. Pediatr. Exerc. Sci. 12:112-127, 2000.

26. Wood, L.E., S. Dixon, C. Grant, and N. Armstrong. Elbow flexion and extension strength relative to body or muscle size in children. Med. Sci. Sports Exerc. 36:19771984, 2004.

27. Wood, J.E., S.G. Meek, and S.C. Jacobsen. Quantitation of human shoulder anatomy for prosthetic arm control-1. Surface modelling. J. Biomech. 22:273-292, 1989.

28. Wretenberg, P., G. Németh, M. Lamontagne, and B. Lundin. Passive knee muscle moment arms measured in vivo with MRI. Clin. Biomech. 11:439-446, 1996. 